\title{
A basic lock-in amplifier experiment for the undergraduate laboratory
}

\author{
K. G. Libbrecht, ${ }^{\text {a) }}$ E. D. Black, and C. M. Hirata \\ Norman Bridge Laboratory of Physics, California Institute of Technology 264-33, Pasadena, California 91125
}

(Received 9 August 2002; accepted 9 April 2003)

\begin{abstract}
We describe a basic experiment for the undergraduate laboratory that demonstrates aspects of both the science and the art of precision electronic measurements. The essence of the experiment is to measure the resistance of a small length of brass wire to high accuracy using a simple voltage divider and a lock-in amplifier. By performing the measurement at different frequencies and different drive currents, one observes various random noise sources and systematic measurement effects. (C) 2003 American Association of Physics Teachers.
\end{abstract}

[DOI: $10.1119 / 1.1579497]$

\section{INTRODUCTION}

Precision electronic measurements are ubiquitous in modern physics. Experimental physicists are frequently faced with the challenge of measuring extremely small electronic signals from any number of sources. Given the widespread use of precision measurement techniques, we feel some obligation to teach at least some of the tools and tricks of this trade. We present here an undergraduate laboratory experiment that describes how the lock-in amplifier can be used to make precision measurements. The lock-in is an extremely versatile measurement tool, and the ideas behind lock-in detection are often used in experimental physics. Our experiment is relatively simple, inexpensive, and robust. Furthermore, it is a hands-on experiment, in which the student is guided through different aspects of the measurement process.

The focus of this experiment is simply to measure the resistance of a short length of brass wire. The resistance of our sample is about $80 \mathrm{~m} \Omega$, small enough that a typical hand-held digital multimeter is not up to the task. With a lock-in, however, the absolute resistance can be measured to within a fraction of a percent fairly easily. We like to point out that although measuring the resistance of a piece of wire is hardly cutting-edge physics, measuring the resistance of a nanotube, nanocontact, or some other small object is. For example, one can imagine that the wire would actually be 20 $\mathrm{nm}$ in diameter and $100 \mathrm{~nm}$ long, chilled to $50 \mathrm{mK}$, and be located at the bottom of an expensive cryostat. Given a maximum allowable current density of, say, $1 \mathrm{~A} / \mathrm{cm}^{2}$, we might be restricted to using only a few $\mathrm{pA}$ in our nanoscale experiment. We do the experiment with a simple wire described here because it is cheaper and it is nearly indestructible. Additionally, the wire measurement demonstrates nicely many noise sources and systematic errors that are often associated with precision electronic measurements. In addition to its intrinsic value, the experiment serves as a prerequisite for more advanced experiments that require lock-in detection.

\section{BACKGROUND: LOCK-IN MEASUREMENT TECHNIQUES}

We first describe the basic measurement techniques used for lock-in detection and signal measurement. ${ }^{1-3}$ A lock-in amplifier is typically used when one has a small signal buried in noise. Consider an experiment in which we wish to measure the response of a system to some stimulus, and we know that the response is very weak. Furthermore, the output of the system, which is typically converted to an electronic signal, contains a lot of electronic noise that we cannot get rid of. Put in terms of voltages, our output signal looks like

$$
V_{\text {signal }}(t)=V_{0}+V_{\text {noise }}(t)
$$

where $V_{0}$ is the response of the system we want to measure-here assumed to be constant in time-and $V_{\text {noise }}(t)$ is noise.

How we respond to this situation depends to some degree on the character of the noise $V_{\text {noise }}(t)$. In some cases the noise we are faced with is pure white noise, also called Gaussian noise. For this type of noise, $V_{\text {noise }}(t)$ fluctuates randomly and rapidly with time in such a way that the statistics of the noise are independent of time, and each value of $V_{\text {noise }}(t)$ is completely uncorrelated with $V_{\text {noise }}\left(t^{\prime}\right)$, provided that $\left|t-t^{\prime}\right|$ is greater than some correlation time $\tau$. A typical picture of white noise as a function of time is shown in Fig. 1. The power spectrum of white noise is independent of frequency up to frequencies $f \approx \tau^{-1}$; at higher frequencies the noise power typically goes to zero. With pure white noise our best recourse is to simply average the output signal with respect to time. White noise has zero expectation value, that is, $\left\langle V_{\text {noise }}\right\rangle=0$, and by time-averaging we obtain $\left\langle V_{\text {signal }}(t)\right\rangle$ $=\left\langle V_{0}+V_{\text {noise }}(t)\right\rangle=\left\langle V_{0}\right\rangle+\left\langle V_{\text {noise }}(t)\right\rangle \rightarrow V_{0}$, so we will get the answer we seek to high accuracy if we average for a long enough time.

In the real world, however, noise is seldom white. Amplifiers and other noise-generating elements often drift slowly with time. Over short periods the output of a typical amplifier will drift a small amount, and over long times it will drift a larger amount. Some of this comes from environmental effects-temperature drifts in the lab, creep in materials, etc., but some is intrinsic to many electronic devices. Detailed studies of many different types of electronic noise have shown that the frequency spectrum of these kinds of noise sources is often approximately proportional to $1 / f$, where $f$ is the frequency. Many electronic devices exhibit this " $1 / f$ noise" even at very low frequencies. After much study there is still considerable debate over just what causes $1 / f$ noise in many devices, but it certainly exists and is almost ubiquitous in amplifiers and other analog electronic devices. Figure 1 shows some typical $1 / f$ noise in comparison with white noise.

In addition to white noise and $1 / f$ noise, most detectors also provide some dc offsets to go along with whatever sig- 

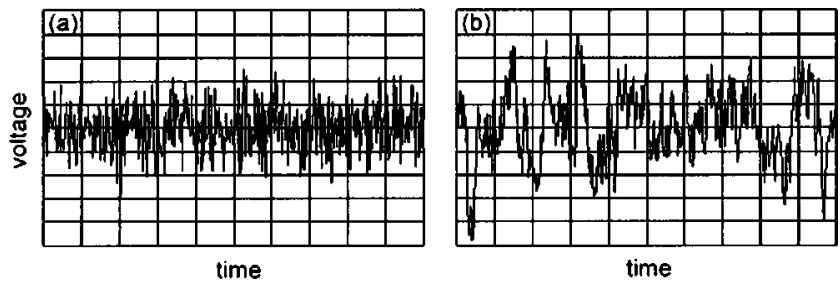

Fig. 1. The left panel (a) shows a signal as a function of time that is dominated by white noise, also called Gaussian noise. White noise has zero expectation value and averages to zero with time. The right panel (b) shows a signal dominated by $1 / f$ noise, which is commonly seen in physics experiments. $1 / f$ noise can be thought of as containing low-frequency drifts which do not average to zero with time (simulated noise plots from MilottiRef. 4).

nal one would like to measure. Including these terms, we see that a somewhat more realistic picture for the signal voltage would be

$$
V_{\text {signal }}(t)=V_{0}+V_{\text {offset }}+V_{\text {white noise }}(t)+V_{1 / f \text { noise }}(t) .
$$

Even if we could remove $V_{\text {offset }}$, at low enough frequencies the noise will still be dominated by $1 / f$ noise, and then a simple time-average is not going to work very well. A long time average will reduce the high-frequency noise contributions, but the longer we average, the more the low-frequency drifts inherent in $1 / f$ noise will contaminate the signal. Once we are dominated by $1 / f$ noise or voltage offsets, signal averaging will not be an effective way to improve our determination of $V_{0}$.

The situation is improved if we can control the signal voltage $V_{0}$. A particularly powerful trick is to chop the signal on and off at some high frequency and take the difference $V_{\text {on }}-V_{\text {off }}$. It is easy to see that any voltage offsets disappear from this difference voltage, and low-frequency drifts make little contribution as well; only higher-frequency noise contributes to the difference signal. If we can also average the difference signal over time, then we will be left with only a noise contribution at the chopping frequency. If the signal voltage is being digitized as a function of time, then it is a simple matter to perform the difference-and-average procedure in software; one just has to include a data flag to keep track of when the signal is on and when it is off. But another route is to use a lock-in amplifier to perform this task. A lock-in is a general purpose piece of laboratory equipment that can be adapted very quickly to different experiments.

Most modern lock-in amplifiers combine analog and digital electronics techniques. The input signal is first amplified and possibly filtered to remove noise above and below the reference frequency, and the resulting signal is then digitized. Lock-in amplifiers typically include a robust, well-behaved, low-noise preamplifier together with a set of electronic filters into which the signal is fed. Sometimes the signal filtering is done with analog electronic filters, and sometimes it is done digitally; the method depends on the particular lock-in used, but it is usually transparent to the user.

In addition to the signal input, one also needs to provide a reference input, which contains a waveform with a strong component at the frequency at which you are modulating the signal. The lock-in electronics then "locks" onto this reference signal and thus determines the operating frequency. A good lock-in can often extract a stable reference signal from a weak reference input, but more typically one presents the lock-in with a square wave of several-volt amplitude that provides an unmistakable reference. Note that the reference signal contains not only a frequency but also a phase, and the latter is often very relevant to a given experiment. (The reference amplitude is irrelevant in principle.)

With a locked reference and a pre-processed input signal, the lock-in then manipulates the signals digitally to produce the desired output. Typically, the reference signal is converted into a sine wave with some adjustable phase, then multiplied by the signal input, and finally averaged to form

$$
V_{\text {out }}=\left\langle V_{\text {signal }}(t) \cos (\omega t+\varphi)\right\rangle,
$$

where the average is a running time average. This procedure picks out one Fourier component of the input signal $V_{\text {signal }}(t)$. If the signal we wish to measure is chopped, then the lock-in will pick out the first Fourier component of the square wave. In the experiment described below, our signal is sinusoidal in nature. Note that the exact method for generating $V_{\text {out }}$, particularly the way the time average is done, varies among lock-in amplifiers, so the expression above is only accurate up to a constant of order unity. Needless to say, these details are provided in the lock-in manual, although it is straightforward to send in a known signal to measure the proportionality constant directly.

A dual-phase lock-in, which is especially useful for some experiments, produces two outputs, the in-phase and quadrature outputs, given by

$$
V_{X}=\left\langle V_{\text {signal }}(t) \cos (\omega t+\varphi)\right\rangle
$$

and

$$
V_{Y}=\left\langle V_{\text {signal }}(t) \sin (\omega t+\varphi)\right\rangle,
$$

respectively, where $\varphi$ is a parameter that one sets on the front panel of the lock-in. The lock-in can also be set to convert these signals digitally to amplitude, $V_{R}$, and phase, $\Phi$, given, respectively, by

$$
V_{R}=\left(V_{X}^{2}+V_{Y}^{2}\right)^{1 / 2}
$$

and

$$
\Phi=\tan ^{-1}\left(V_{Y} / V_{X}\right) .
$$

Both representations are useful, of course, depending on what kind of signal is being examined.

To see what a flexible instrument the lock-in amplifier is, consider the experiment shown in Fig. 2. Here the goal is to observe fluorescence from a sample illuminated by a laser. The laser beam is chopped using a mechanical chopping wheel, thus causing the fluorescence to turn on and off at the chopping frequency. The first thing you gain by using a lock-in for this experiment is flexibility. The lock-in has a very low-noise input amplifier, and the sensitivity can be adjusted over many orders of magnitude. Thus just about any input signal can be seen with a lock-in-this is very useful when one is frequently changing samples or laser parameters.

The second thing you gain is some freedom from worrying about ambient light getting into your detector. With the chopper working at $1 \mathrm{kHz}$ or so, the lock-in can almost completely reject the dc and 60/120-Hz signals coming from ambient lights, provided these sources don't swamp either the detector or the lock-in (since both will have limited dynamic range). Finally, problems with voltage offsets and $1 / f$ noise in the detector are also minimized using a lock-in. 


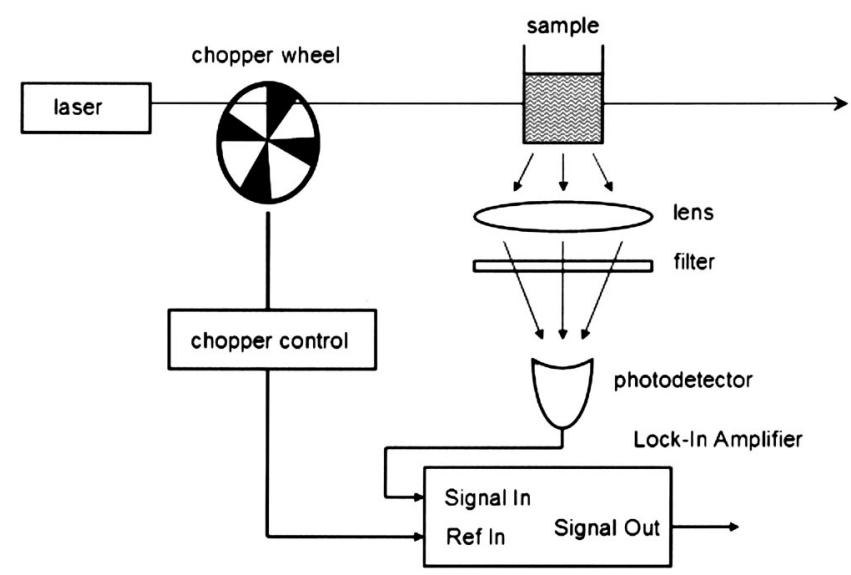

Fig. 2. A typical lock-in amplifier application in which one measures the fluorescence from a sample that is illuminated by a chopped laser beamRef. 5.

A popular lock-in demonstration experiment, shown in Fig. 3, is to modulate an LED using a simple square wave, say at $1 \mathrm{kHz}$, and examine the light output using a photodiode. With the LED close to the photodiode the signal can be seen clearly on an oscilloscope, and thus can be measured directly. With larger separations the photodiode signal becomes much weaker, to the point that it cannot be seen at all on the oscilloscope trace. With the lock-in detector, however, the signal remains strong at the lock-in output even after the photodiode signal appears to be swamped with noise from the ambient lighting.

\section{MORE BACKGROUND: NOISE SPECTRAL DENSITY}

For any physically meaningful noise we can define a new "smoothed" noise function $V_{\text {noise, } \tau}(t)$ that is a running timeaverage of the noise

$$
V_{\text {noise, } \tau}(t)=\frac{1}{\tau} \int_{t^{\prime}=t}^{t^{\prime}=t+\tau} V_{\text {noise }}\left(t^{\prime}\right) d t^{\prime} .
$$

For white noise we have that $\left\langle V_{\text {noise, } \tau}(t)\right\rangle=0$ and $\left\langle V_{\text {noise }, \tau}(t)^{2}\right\rangle^{1 / 2} \equiv \sigma_{\text {noise, } \tau}$ is some constant (measured in units of volts). Physically this is a reasonable definition because we never actually measure the noise voltage at an instant in time, but rather we are always averaging over some short

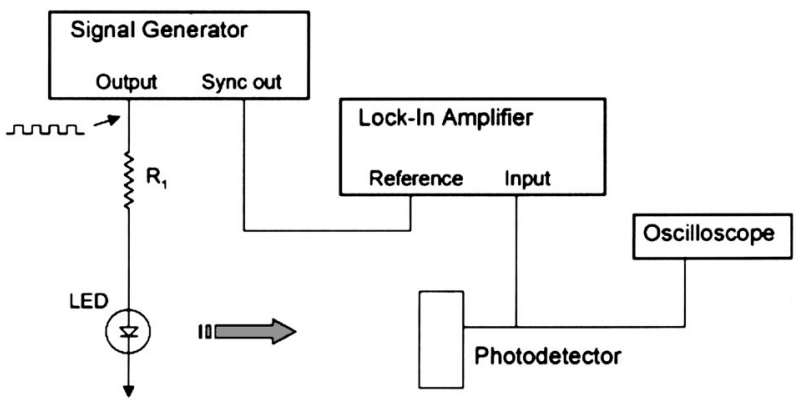

Fig. 3. A popular experiment to demonstrate how lock-in detection can recover a small signal buried in noise. When the LED is close to the photodetector, the signal is large and can be seen directly on the oscilloscope. When the LED is farther away, the signal is no longer visible on the oscilloscope, but is easily detected with the lock-in. time $\tau$. What we see plotted in Fig. 1 is not $V_{\text {noise }}(t)$, but rather $V_{\text {noise, } \tau}(t)$. Naturally $V_{\text {noise }, \tau}(t)$ depends on the averaging time $\tau$, the longer the averaging time $\tau$, the smaller $\sigma_{\text {noise }, \tau}$ and $V_{\text {noise, } \tau}(t)$ will be, and for white noise $\sigma_{\text {noise, } \tau}$ $\sim \tau^{-1 / 2}$. ${ }^{6}$ We see that the averaging time $\tau$ is effectively equivalent to the noise correlation time mentioned above.

In the same way that measuring noise in the time domain always involves some averaging time $\tau$, measuring the noise power spectrum always involves an average over a range of frequencies, called the measurement bandwidth (typically stated in $\mathrm{Hz}$ ). If we compute the noise power spectrum using measurements over a finite time $T_{\text {ave }}$, then for white noise we find

$$
\widetilde{P}_{\text {noise }, B}(f)=\left|\frac{1}{T_{\text {ave }}} \int V_{\text {noise }}(t) e^{i 2 \pi f t} d t\right|^{2}
$$

and

$$
\begin{aligned}
\left\langle\widetilde{P}_{\text {noise }, B}(f)\right\rangle & =\frac{1}{T_{\text {ave }}^{2}}\left|\sum_{j=1}^{j=N=T_{\text {ave }} / \tau} \sigma_{\text {noise }, \tau}\left(t_{j}\right) e^{i 2 \pi f t} \tau\right|^{2} \\
& =\frac{1}{T_{\text {ave }}^{2}} N \sigma_{\text {noise }, \tau}^{2} \tau^{2}=\frac{\sigma_{\text {noise }, \tau}^{2} \tau}{T_{\text {ave }}}=\sigma_{\text {noise }, \tau}^{2} \tau B,
\end{aligned}
$$

where $B=1 / T_{\text {ave }}$ is the measurement bandwidth. The sum was evaluated knowing that the noise is uncorrelated over times greater than $\tau$. Put another way, $V_{\text {noise }}(t)$ exhibits no long-range correlations, hence on long time scales the integral undergoes a random walk with a mean-squared value proportional to $T_{\text {ave }}$.

Since $\sigma_{\text {noise, } \tau} \sim \tau^{-1 / 2}$ we see that $\left\langle\widetilde{P}_{\text {noise }, B}(f)\right\rangle$ is equal to some constant times the bandwidth $B$. We therefore define the bandwidth-independent power spectral density

$$
\begin{aligned}
S(f) & =\lim _{T_{\text {ave }} \rightarrow \infty} \frac{1}{T_{\text {ave }}}\left|\int V_{\text {noise }}(t) e^{i 2 \pi f t} d t\right|^{2} \\
& =\widetilde{P}_{\text {noise }, B}(f) / B .
\end{aligned}
$$

We see that $S(f)$ is a well-defined function that depends on the intrinsic noise in the system as a function of frequency. $S(f)^{1 / 2}$ has the dimensions of $\mathrm{V} / \sqrt{\mathrm{Hz}}$ (called "volts per root hertz"), and this function is typically all one needs to know about the random noise in a signal. For pure white noise $S(f)^{1 / 2}$ is equal to a constant, while for $1 / f$ noise $S(f)^{1 / 2}$ $\sim 1 / f$.

By comparing Eqs. (1) and (2) we see that $\left[\widetilde{P}_{\text {noise }, B}(f)\right]^{1 / 2}$ is precisely what the lock-in amplifier measures in the absence of any signal. Thus the fluctuations in the lock-in output $V_{\text {out }}$ will be proportional to the power spectral density at the reference frequency, $S(f)^{1 / 2}$, times $B^{1 / 2}$, the square root of the bandwidth of the measurement. Note that $B$ $=\beta / T_{\text {filter }}$ is the equivalent noise bandwidth when using a lock-in, where $T_{\text {filter }}$ is the averaging time indicated by the lock-in, and $\beta$ depends on how the signal averaging is performed (which is described in detail in the manual). Thus we see that the noise in a lock-in measurement will go like $T_{\text {filter }}^{-1 / 2}$, as we would expect.

One source of noise in our measurement, which the manufacturer has worked hard to reduce, is the input amplifiers inside the lock-in. For example, our lock-in manual states the input noise is no more than $6 \mathrm{nV} / \sqrt{\mathrm{Hz}}$ at $1 \mathrm{kHz}$. Thus if our 


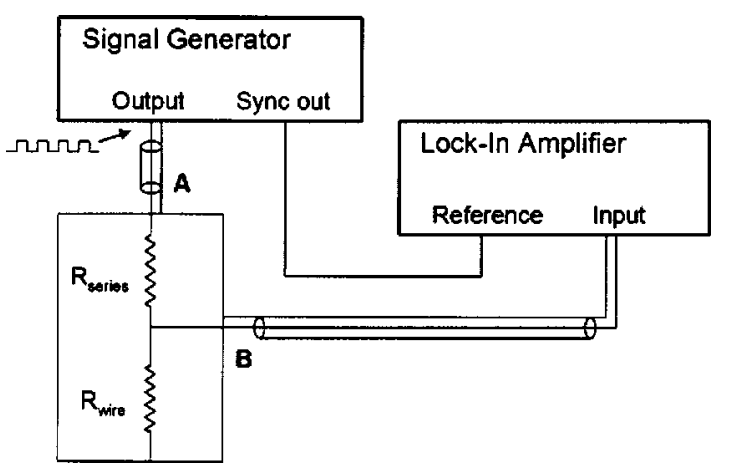

Fig. 4. The circuit used to measure the resistance $R_{\text {wire }}$. The two resistors are soldered together and placed into a small box; the rest of the connections are made using coaxial cables.

reference is at $1 \mathrm{kHz}$ and we short the lock-in input with some small resistor (so the input signal is zero), then the signal we measure would have an effective noise of $6 \mathrm{nV} / \sqrt{\mathrm{Hz}}$. Therefore, if we integrate for $10 \mathrm{~s}$, the output display should display rms fluctuations of about $6 / \sqrt{10} \mathrm{nV}$ $\approx 2 \mathrm{nV}$.

Resistors can introduce noise from thermal fluctuations into a measurement. This is called resistor thermal noise, or Johnson noise, and the rms amplitude of the thermal noise voltage is given by

$$
V_{\text {rms,thermal }}=\sqrt{4 k T R B} \quad(\mathrm{~V}),
$$

or in terms of spectral density

$$
\begin{aligned}
\left.V_{\text {rms,thermal }} \text { spectral density }\right) & =\sqrt{4 k T R} \quad(\mathrm{~V} / \sqrt{\mathrm{Hz}}) \\
& =0.128 \sqrt{\frac{R}{1 \Omega}} \quad(\mathrm{nV} / \sqrt{\mathrm{Hz}}),
\end{aligned}
$$

where $R$ is the resistance value and $B$ is the bandwidth of measurement.

\section{MEASURING A RESISTOR}

The objective of the first part of this lab is to measure the resistance of a short length of brass wire. Specifically, we use an unknown alloy wire with a length of $17 \mathrm{~cm}$ and a diameter of $0.4 \mathrm{~mm}$ that gives a resistance of about $80 \mathrm{~m} \Omega$. There are essentially two ways to measure the electrical resistance of any device-one can either send a known current through it and measure the resulting voltage across it, or apply a known voltage and measure the resulting current through it. In most cases, including our case, the former option is technically easier. We use the simple resistor divider circuit shown in Fig. 4 with $R_{\text {series }}=1 \mathrm{k} \Omega$. The current in this case is $I=V_{A} /\left(R_{\text {series }}+R_{\text {wire }}\right)$, and the voltages we need to measure are $V_{A}$ and $V_{B}$. The wire resistance can then be computed as

$$
R_{\text {wire }}=\frac{V_{B}}{V_{A}} R_{\text {series }}\left(1+\frac{R_{\text {wire }}}{R_{\text {series }}}\right) \approx \frac{V_{B}}{V_{A}} R_{\text {series }},
$$

where in our case the approximation is accurate to a part in $10^{4}$. We see that our measurement of $R_{\text {wire }}$ can be no more accurate than our knowledge of $R_{\text {series }}$, but since $R_{\text {series }}$ is approximately $1 \mathrm{k} \Omega$ we can measure it fairly accurately with a simple digital multimeter.

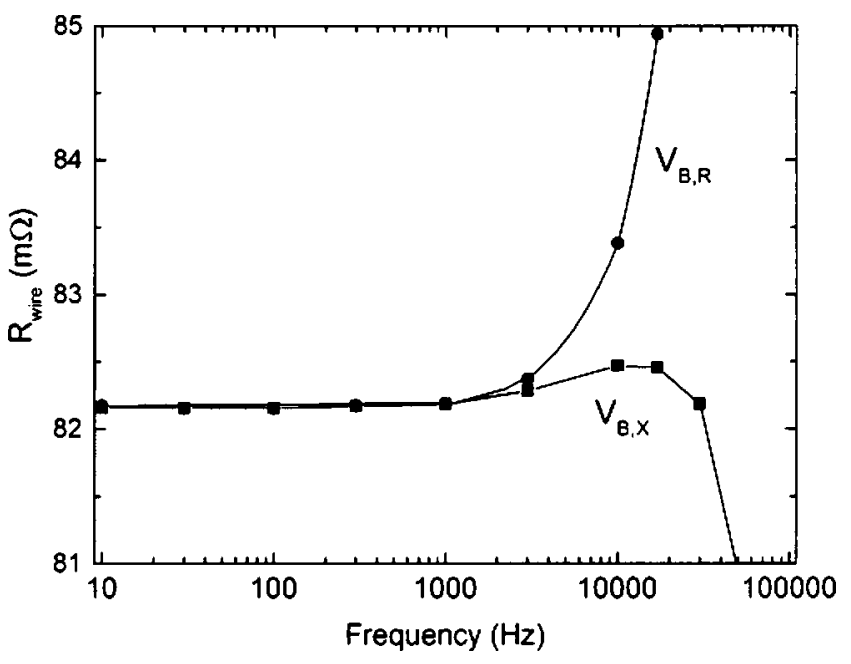

Fig. 5. A measurement of $R_{\text {wire }}$ as a function of the signal generator frequency with a large fixed input voltage. Specifically, $V_{A \text {, set }}$ was a $1-\mathrm{V}$ sine wave, and the lock-in time constant was $3 \mathrm{~s}$. $R_{\text {wire }}$ was determined using either the total signal amplitude $V_{B, R}$ or the in-phase component $V_{B, X}$. This graph demonstrates systematic effects in the measurement that arise from capacitive effects. These effects are reduced by using the in-phase signal $V_{B, X}$, but they are not eliminated.

For our experiment we use a Stanford Research Systems model SR830 DSP lock-in amplifier, and a Berkeley Nucleonics Corporation Model 625 signal generator is used to generate a sine-wave signal. The circuit configuration in Fig. 4 was used to measure $V_{B}$, and the signal generator was connected directly to the lock-in input to measure $V_{A}$. From these voltages $R_{\text {wire }}$ was determined as a function of $V_{A}$ and the signal generator frequency $\nu$. [One important feature of the circuit that is not shown in Fig. 4 is a 4:1 voltage divider that was inserted between the signal generator sync output and the lock-in reference input. This divider reduced the level of the sync signal by a factor of 4 before it went into the lock-in amplifier. In principle, this divider should do nothing; however, before this divider was inserted (i.e., when the sync out was fed directly into the lock-in reference input) we found a small but significant offset in the lock-in reading. We do not understand the origin of this problem, but it appears to be due to cross-talk inside the lock-in amplifier. The divider seemed to eliminate this problem completely.]

We want to convey an important lesson: all precision measurements are limited by both random noise and systematic effects. Much of the art of experimental physics is dealing with these problems. To demonstrate the kinds of systematic errors that can be present, we first measure $R_{\text {wire }}$ as a function of $\nu$ with the signal generator output set point, $V_{A \text {, set }}$ fixed. (Our signal generator is digital, so $V_{A \text {, set }}$ is a numerical input value; however, we always measure $V_{A}$ directly using the lock-in.) We measure $R_{\text {wire }}$ two ways: using the measured signal amplitude $V_{B, R}$, and using the in-phase component $V_{B, X}$ (recall the definition $V_{R}^{2}=V_{X}^{2}+V_{Y}^{2}$ given above). The lock-in phase is adjusted so that the direct measurement of $V_{A}$ gives $V_{Y}=0$; that way, we expect $V_{B, Y}=0$ if our resistors have purely real impedances. (In our case the phase offset between the signal generator output and the sync out was measured to be $2.3^{\circ}$.)

The results of these measurements are shown in Fig. 5. Note that we used a large $V_{A \text {, set }}$ and a long time constant $\tau_{0}$, 


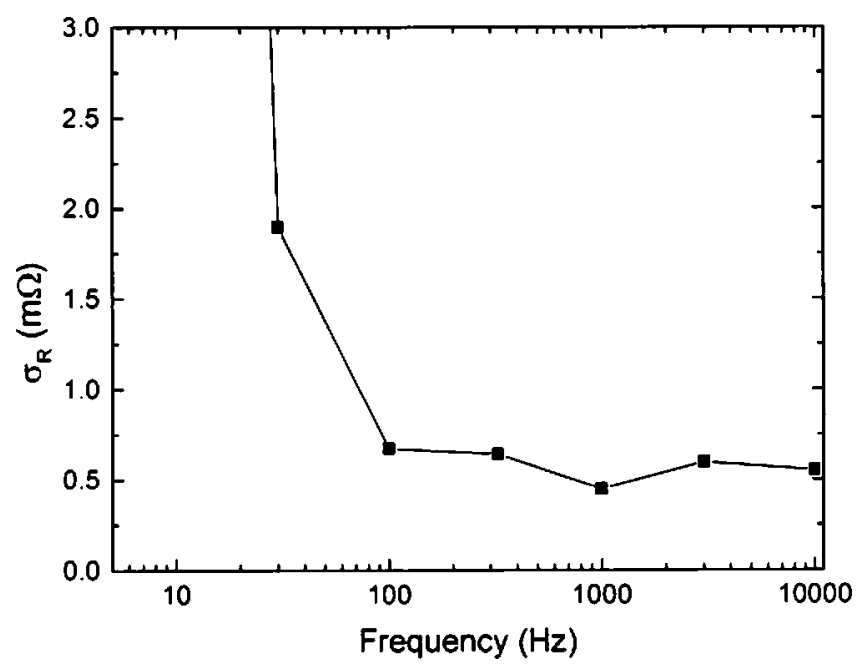

Fig. 6. The random noise in a single measurement of $R_{\text {wire }}$ made with a small input voltage and a small lock-in time constant. Specifically, $V_{A \text {, set }}$ was a sine wave of $10-\mathrm{mV}$ amplitude, and the measurement was made using the in-phase component $V_{B, X}$ and a lock-in time constant of $0.3 \mathrm{~s}$. This demonstrates how the noise increases sharply when the measurement is done at low frequencies.

so the random noise in these measurements is small. We first see that our measurements of $R_{\text {wire }}$ based on $V_{B, R}$ are constant at low frequencies, but increase rapidly above about 3 $\mathrm{kHz}$. The frequency dependence comes mainly from capacitive effects - the cable capacitance and the stray capacitance couple with the finite output impedance of the signal generator to produce a complex impedance. The interested student is invited to investigate these effects by changing cable lengths and by adding a small series resistor between the signal generator and $R_{\text {series }}$ (thus changing the effective signal generator output impedance). The hands-on nature of this simple experiment makes this kind of investigation easy to perform although we don't require it as part of the lab. The main lesson from the graph in Fig. 5 is that the real circuit is not necessarily the same as the ideal circuit drawn in Fig. 4. The power of lock-in detection is that one can simply measure $R_{\text {wire }}$ as a function of $\nu$ to see the magnitude of these systematic effects directly.

If the systematic effects were entirely capacitive, we would expect to obtain a more accurate measurement of $R_{\text {wire }}$ using $V_{B, X}$, the in-phase component of $V_{B}$, because the capacitive impedances are purely complex. We see in Fig. 5 that this is indeed the case. Using $V_{B, X}$, one finds that the systematic errors become very large only above about 30 $\mathrm{kHz}$, where they are a factor of 10 higher than what one obtains using $V_{B, R}$. At very high frequencies we see that the circuit in Fig. 4 no longer represents the real circuit well at all, so our determination of $R_{\text {wire }}$ would have to proceed differently at high frequencies.

Another lesson we wish to convey in this lab is that random errors are also frequency dependent owing to $1 / f$ noise and other effects. To demonstrate this we again measure $R_{\text {wire }}$ as a function of frequency, but this time with a small $V_{A \text {,set }}$ and a shorter time constant $\tau_{0}$. The random errors are estimated simply by recording 16 separate measurements of $V_{B, X}$, the measurements separated by several $\tau_{0}$. The standard deviation $\sigma_{R}$ was then determined from these 16 samples. The results are shown in Fig. 6. We see that $\sigma_{R}$ is

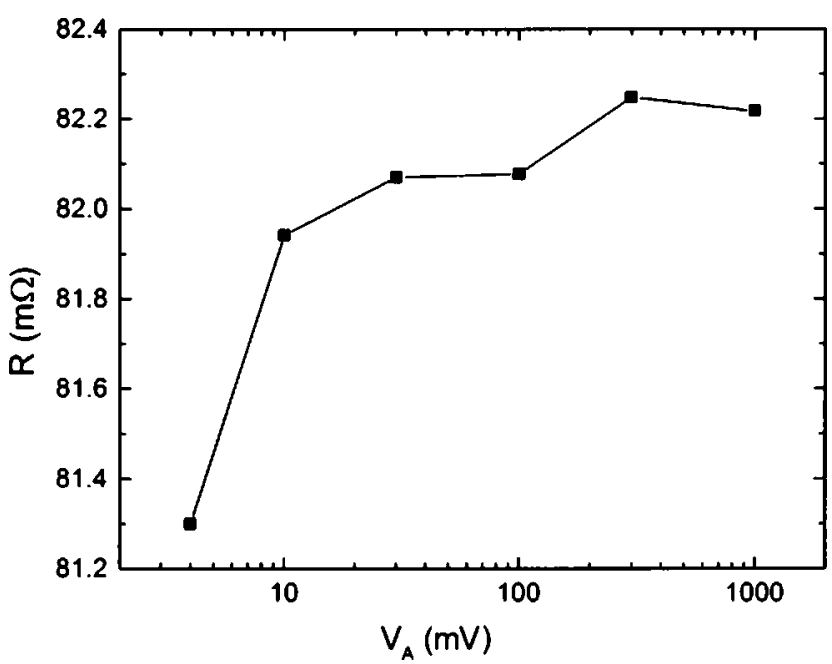

Fig. 7. Measurements of $R_{\text {wire }}$ as a function of the input voltage $V_{A}$. The frequency is fixed at $\nu=1 \mathrm{kHz}$. The measurement was made using $V_{B, X}$ data and a lock-in time constant of $0.3 \mathrm{~s}$. Again we see how systematic errors dominate the uncertainty, particularly when the signal level is low.

much higher at low frequencies, probably due to the intrinsic noise in the lock-in amplifier. The curious student is invited to compare these measurements with the noise specifications given in the lock-in manual. We note by comparing Figs. 5 and 6 that there is an optimal frequency for making measurements of $R_{\text {wire }}$. If $\nu$ is too high, systematic errors become problematic; if $\nu$ is too low, the random noise is greater. An interesting side point is that the measurement errors were huge at $\nu=300 \mathrm{~Hz}$ when $V_{A}$ was small, because of $60-\mathrm{Hz}$ noise harmonics. This problem was eliminated by measuring at $\nu=325 \mathrm{~Hz}$ instead of $300 \mathrm{~Hz}$.

Next we ask students to determine just what $R_{\text {wire }}$ really is, and how well it can be measured if we are restricted to passing only very small currents through the wire. To this end we measure $R_{\text {wire }}$ as a function of $V_{A \text {,set }}$, with the frequency fixed at $\nu=1 \mathrm{kHz}$. We use a long time constant to reduce the random fluctuations in $V_{B}$. The results are shown in Fig. 7. Again we see a systematic trend with $V_{A}$, which is probably due to some unexpected signal coupling, either in our circuit or in the lock-in itself. With $V_{A}=4 \mathrm{mV}$ we find that $V_{B}$ is only $300 \mathrm{nV}$, so there are any number of stray voltage effects that could produce the necessary offset. The interested stu-

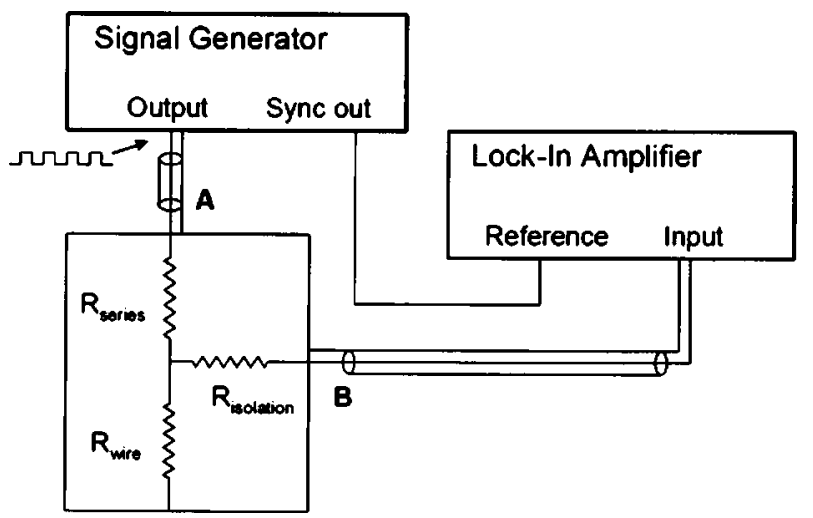

Fig. 8. A circuit schematic for measuring $R_{\text {wire }}$ in the presence of an isolation resistor. By varying $R_{\text {isolation }}$ the Johnson noise can be measured. 
dent is invited to try and track this down. In the end we see from Fig. 7 that $R_{\text {wire }} \approx 82.2 \pm 0.2 \mathrm{~m} \Omega$, with the uncertainty coming mainly from systematic effects. An absolute accuracy of $0.25 \%$ is not too bad for such a small resistor, using such a simple circuit. This level of accuracy is sufficient to see the change in $R_{\text {wire }}$ when it is warmed slightly, by cupping one's hands around the wire or by blowing on it slightly.

Finally, we use the circuit in Fig. 8 to demonstrate the effects of Johnson noise. This circuit is motivated by imagining that our sample is sitting at the bottom of a cryostat at $100 \mathrm{mK}$. In this case we cannot connect wires to it directly because of the heat load, and therefore $R_{\text {isolation }}$ cannot be made too small. By measuring $\sigma_{R}$ again with different values for $R_{\text {isolation }}$, Johnson noise can be observed.

\section{DISCUSSION}

The purpose of this laboratory experiment is to introduce students to the science and art of precision electronic measurements. This hands-on lab demonstrates the concepts of lock-in detection, noise spectral density, and the trade-offs between random and systematic errors. We use the lab as a prerequisite for other labs requiring the use of lock-in detection for small-signal measurements. We believe this lab also reinforces the point that precision measurements can be tricky, hence varying whatever measurement parameters one can vary (in this case $V_{A}$ and $\nu$ ) is good experimental practice.

\footnotetext{
${ }^{a)}$ Electronic mail: $\mathrm{kgl} @$ caltech.edu

${ }^{1}$ P. Horowitz and W. Hill, The Art of Electronics (Cambridge U.P., New York, 1989), 2nd ed., p. 1031.

${ }^{2}$ D. W. Preston and E. R. Dietz, The Art of Experimental Physics (Wiley, New York, 1991), p. 367.

${ }^{3}$ R. Wolfson, Am. J. Phys. 59, 569 (1991).

${ }^{4}$ Simulated noise plots from Edoardo Milotti, at http://www.fisica.uniud.it/ milotti/Research/loverf/loverf_noise.html (2002) (with permission).

${ }^{5}$ Graph adapted from http://www.chem.vt.edu/chem-ed/electronics/instrum/ lock-in.html.

${ }^{6}$ D. T. Gillespie, Am. J. Phys. 64, 225 (1996); 61, 1077 (1993).
}

\section{AMERICAN JOURNAL OF PHYSICS ON THE INTERNET}

For access to the online version of AJP, go to AIP's Online Journal Publishing Service: http://aapt.org/ajp.

Browsing abstracts and tables of contents of online issues (beginning with January 1999) and searching of titles, abstracts, etc., back to 1975 is unrestricted.

Institutional and library ("nonmember") subscribers have access via IP addresses to the full text of articles that are online; to activate access, these subscribers should contact AIP, Circulation \& Fulfillment Division, 800-344-6902; outside North America 516-576-2270 or subs@aip.org.

Individual ("member") subscribers to the paper version who wish (for an additional fee) to add access to the online version should similarly contact AAPT or go to the AAPT website: http://www.aapt.org/.

AJP's home page at the editorial office (http://www.kzoo.edu/ajp/) contains the Table of Contents of the next month's issue several weeks before publication, the Tables of Contents for the last several years, the "Statement of Editorial Policy," "Information for Contributors," membership rates and a membership application, library ("nonmember") subscription rates, etc. 